\title{
Estudo de perfil de espalhamento usando difração de raios $X$ de amostras de sangue irradiadas
}

\section{Study of X-ray diffraction scattering profiles in irradiated blood samples}

\author{
Nivia G. V. Pinto ${ }^{1}$ \\ Rogério A. Figueiras ${ }^{1}$ \\ Leonardo Mendonça ${ }^{1}$ \\ Delson Braz ${ }^{I}$ \\ Regina C. Barroso ${ }^{2}$ \\ Ricardo T. Lopes ${ }^{1}$
}

\begin{abstract}
O aumento nas aplicações das radiações ionizantes pode produzir exposição de pessoas e objetos a campos de radiação, onde a intensidade depende do tipo da fonte e da atividade desenvolvida. Nesse trabalho, perfis de espalhamento de amostras liofilizadas de plasma, matriz celular e sangue total foram estudados. Os perfis de espalhamento foram obtidos com um difratometro de raios X Shiamadzu (XRD6000) no Laboratório de Instrumentação Nuclear, LIN/Coppe. As amostras foram irradiadas com valores de dose de 2cGy a 100cGy no Inca, no Rio de Janeiro, e todas as amostras de plasma, matriz celular e sangue total foram liofilizadas a $-60^{\circ} \mathrm{C}$ e $780 \mathrm{mmHg}$ até completa eliminação de água. Os ângulos de espalhamento de $5^{\circ}$ a $35^{\circ}$ foram varridos em passos de $0,05^{\circ} \mathrm{com}$ tempo de integração de 3 segundos. Os perfis de espalhamento das amostras irradiadas foram comparados com os perfis de amostras não irradiada. Nesse estudo observou-se que os perfis das amostras de matriz celular e sangue total são bastante parecidos. O perfil de espalhamento do plasma é muito característico porque em adição ao primeiro e ao segundo pico essas amostras mostraram mais dois picos estreitos. Os valores de $\theta_{1}$ e $\theta_{2}$ das amostras de plasma irradiado são maiores que os obtidos pelas amostras de plasma não irradiado e os valores de $\theta_{1}$ e $\theta_{2}$ das amostras de matriz celular irradiada são menores que os obtidos pelas amostras de matriz celular não irradiada. Rev. bras. hematol. hemoter. 2008;30(2):132-135.
\end{abstract}

Palavras-chave: Sangue; difração de raios $X$.

\section{Introdução}

Nas últimas décadas, muitos autores têm realizado medidas de perfis de espalhamento a baixo ângulo (LAXS) de amostras biológicas. ${ }^{1-4} \mathrm{O}$ crescente interesse em tais medidas deve-se à natureza característica do espalhamento coerente da radiação. Dessa forma, LAXS de amostras biológicas, como o sangue e seus componentes, têm importância nos campos da biologia e medicina. Por meio do LAXS entende-se a estrutura molecular da amostra obtendo-se uma assinatura que é característica do material em estudo.

O espalhamento coerente das amostras biológicas é dominado pelo perfil da água, ${ }^{5,6}$ já que os tecidos biológicos são em grande parte compostos desse elemento. A remoção da água por liofilização produz espectros consideravelmente diferentes das amostras não liofilizadas. Essa técnica, também conhecida como desidratação a frio (freeze dry) é um eficiente processo de secagem de amostras biológicas que preserva células, enzimas, vacinas, vírus, soros e derivados sangüíneos. Na liofilização, as amostras biológicas são submetidas a baixa temperatura e pressão, fazendo com que a água presente se transforme em gelo que, em seguida, devido à baixa pressão, sublimará.

A caracterização de amostras liofilizadas (irradiadas e não irradiadas) de sangue total, matriz celular e plasma foi realizada através da obtenção de perfis de espalhamento coerente de raios X. As medidas foram realizadas no Laboratório de Instrumentação Nuclear, LIN/Coppe/UFRJ. Esse trabalho tem por objetivo a compreensão dos perfis de espalhamento coerente de raios $\mathrm{X}$ dos principais componentes

${ }^{1}$ Laboratório de Instrumentação Nuclear (LIN) da Universidade Federal do Rio de Janeiro (UFRJ/Coppe)-RJ.

${ }^{2}$ Universidade do Estado do Rio de Janeiro (Uerj) - Instituto de Física (IF) - Rio de Janeiro-RJ.

Correspondência: Nivia G. Villela Pinto

Centro de Tecnologia, Bloco I, sala 133 - Ilha do Fundão

21949-900 - Rio de Janeiro, RJ - Brasil

Tel: (21) 2562-7311; fax: (21) 2562-8444

E-mail:nivia@lin.ufrj.br 
do sangue irradiados e não-irradiados por meio da sua caracterização. A caracterização também poderá ser útil no estudo de exposição das amostras de sangue a radiações ionizantes de diferentes tipos e energias.

\section{Material e Método}

As amostras de sangue $(n=75)$ foram coletadas de doadores por meio de punção venosa no Laboratório de Análises Clínicas Dr. Elilel Figueiredo, Rio de Janeiro. Após a coleta, as amostras foram acondicionadas em geladeira a uma temperatura de $4^{\circ} \mathrm{C}$. As amostras foram irradiadas com doses que variaram de 2 cGy a 100 cGy, utilizando-se uma bomba de cobalto Theratron $780 \mathrm{C}$ no Instituto Nacional do Câncer, Inca, Rio de Janeiro.

Para realizar a separação do plasma da matriz celular, as amostras ficaram em repouso por uma hora. Devido à gravidade, a matriz celular, mais pesada, se depositou no fundo do tubo, enquanto o plasma, mais leve, ficou sobrenadante. O plasma e a matriz celular foram retirados do tubo por aspiração utilizando-se uma pipeta. As amostras de sangue total, plasma e matriz celular foram, então, liofilizadas a $-60^{\circ} \mathrm{C}$ e $780 \mathrm{mmHg}$, por 48 horas, até a completa eliminação da água e, em seguida, pulverizadas.

As medidas de difração de raios X foram realizadas utilizando-se um difratômetro comercial Shimadzu XRD 6000 no Laboratório de Instrumentação Nuclear, Coppe/UFRJ. As medidas foram realizadas em geometria de reflexão $\theta-2 \theta$ utilizando-se um feixe de raios X de $8,047 \mathrm{keV}(\lambda=1,541 \AA)$. Os perfis foram obtidos no intervalo de $5^{\circ}$ a $35^{\circ}$ com passo angular de $0,05^{\circ}$ a cada 3 segundos. A intensidade da radiação espalhada foi coletada por um detector cintilador de $\mathrm{NaI}$.

\section{Resultados}

As figuras 1 a 3 apresentam os perfis de difração típicos obtidos para as amostras padrão de sangue total (ST), matriz celular (MC) e plasma (PL), respectivamente.

No caso de materiais amorfos, os padrões de difração não produzem picos estreitos como nos materiais cristalinos, pois os arranjos dos átomos são irregulares. ${ }^{7}$ No caso do sangue, os perfis de espalhamento possuem dois picos bastante largos $\left(\theta_{1}\right.$ e $\left.\theta_{2}\right)$ devidos, principalmente, às estruturas de moléculas grandes como, por exemplo, as proteínas. A tabela 1 mostra os valores médios encontrados para $\theta_{1}$ e $\theta_{2}$.

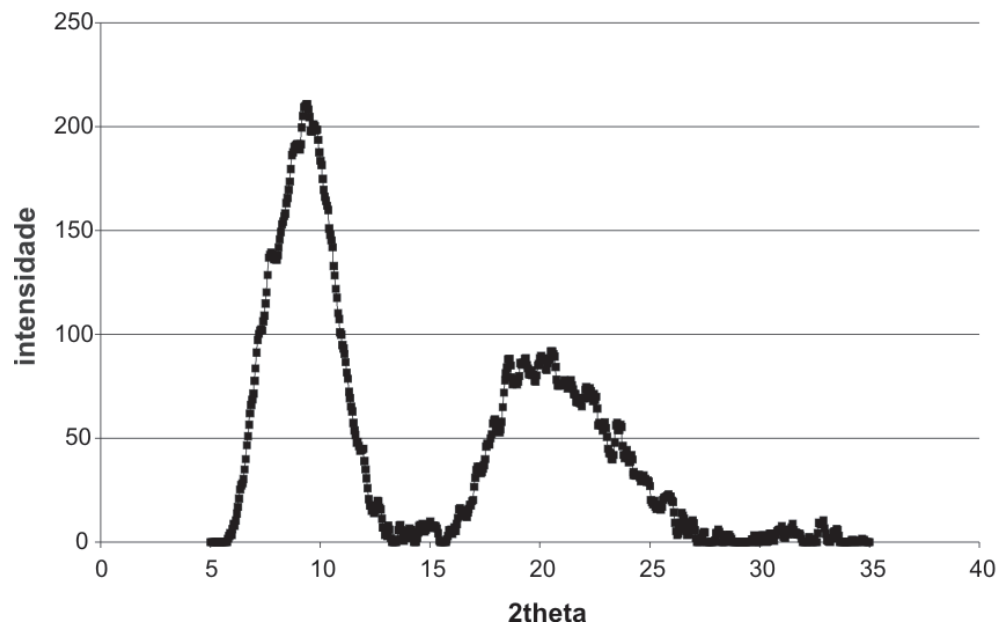

Figura 1. Perfil de espalhamento típico para matriz celular padrão

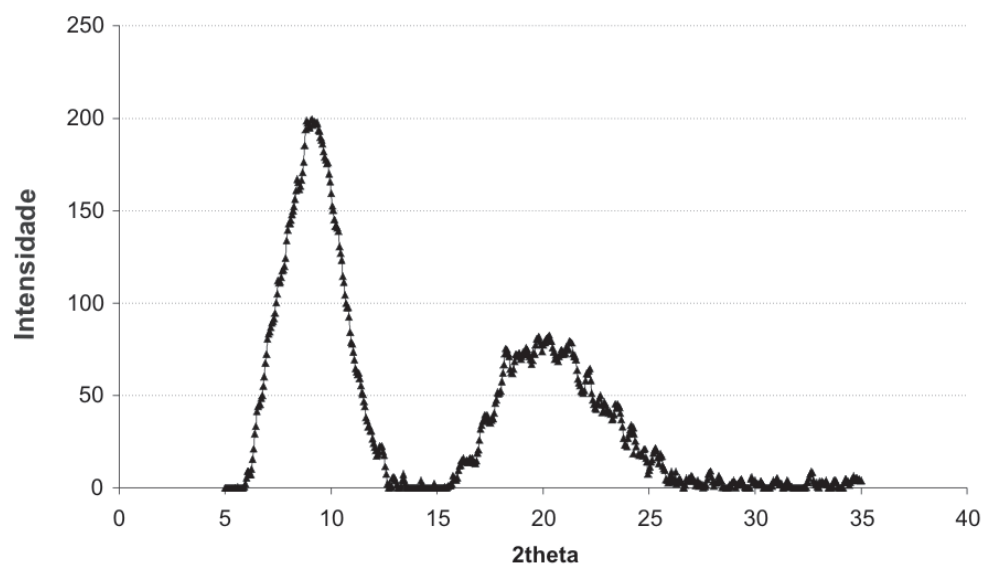

Figura 2. Perfil de espalhamento de sangue total (ST) não irradiada

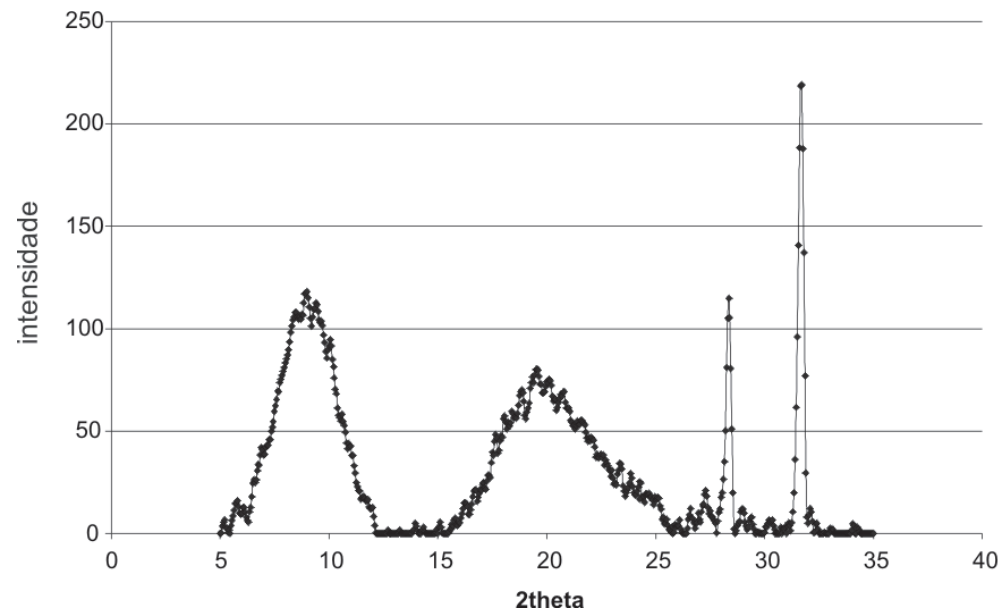

Figura 3. Perfil de espalhamento de plasma (PL) não irradiada 
Tabela 1. Valores médios de $\theta_{1}$ e $\theta_{2}$

\begin{tabular}{lll}
\hline & $\theta_{1}$ (Graus) & $\theta_{2}$ (Graus) \\
\hline ST & $9,30 \pm 0,05$ & $20,08 \pm 0,19$ \\
MC & $9,48 \pm 0,06$ & $20,65 \pm 0,72$ \\
PL & $9,08 \pm 0,16$ & $19,50 \pm 0,60$ \\
\hline
\end{tabular}

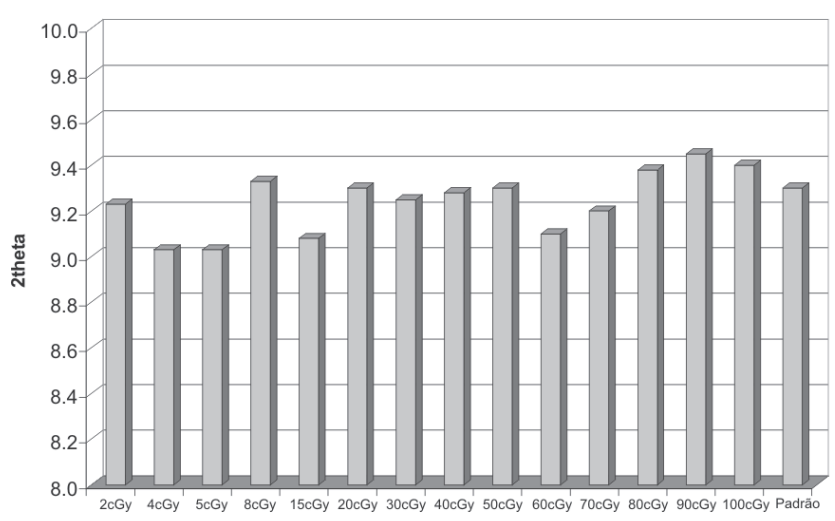

Figura 4. Variação de $\theta_{1}$ em função da dose para as amostras de ST

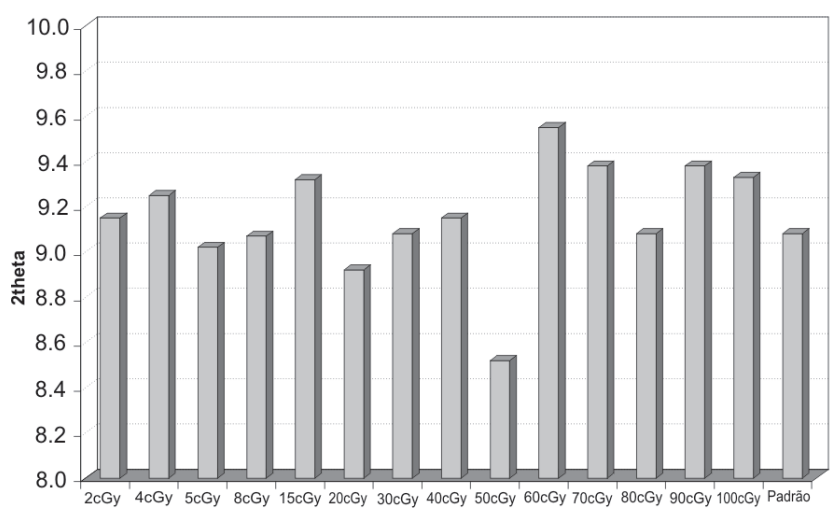

Figura 5. Variação de $\theta_{1}$ em função da dose para as amostras de MC

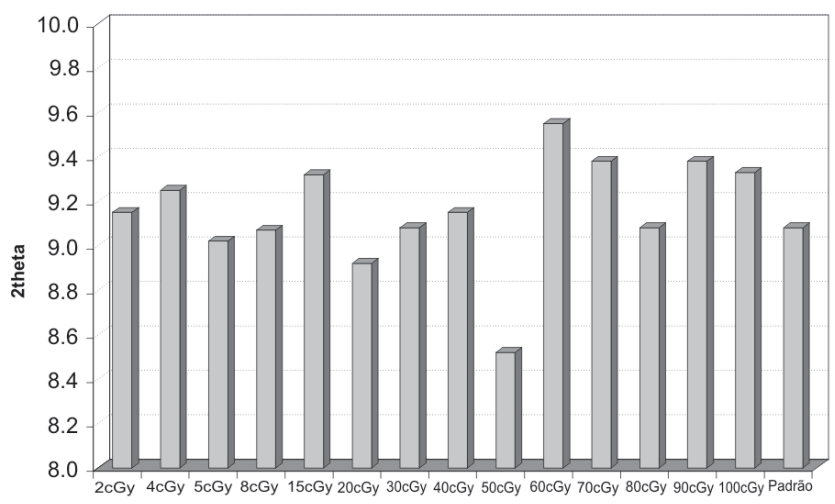

Figura 6. Variação de $\theta_{1}$ em função da dose para as amostras de PL

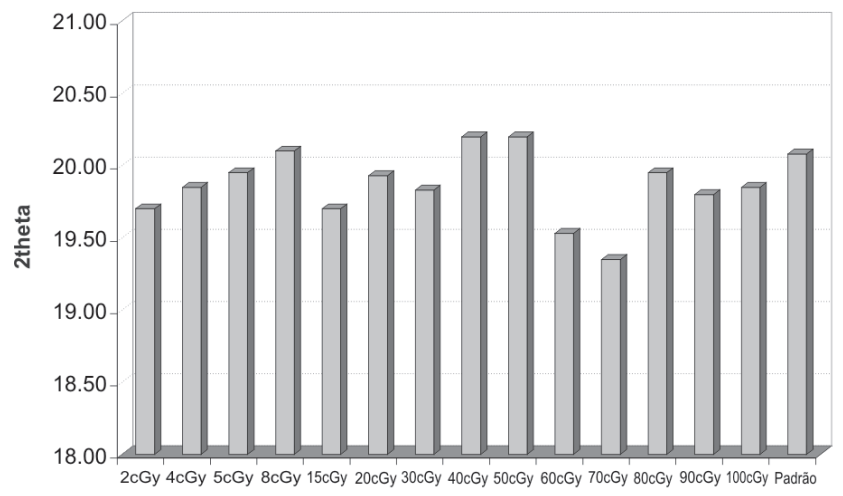

Figura 7. Variação de $\theta_{2}$ em função da dose para as amostras de ST

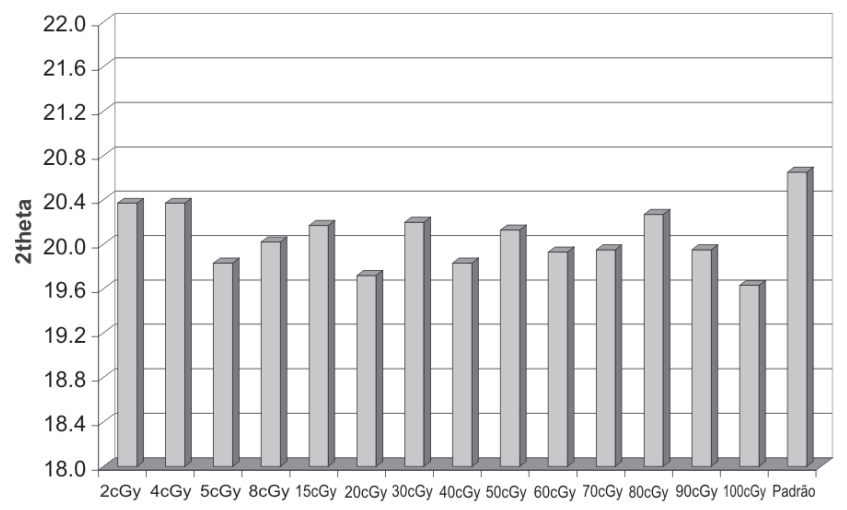

Figura 8. Variação de $\theta_{2}$ em função da dose para as amostras de MC

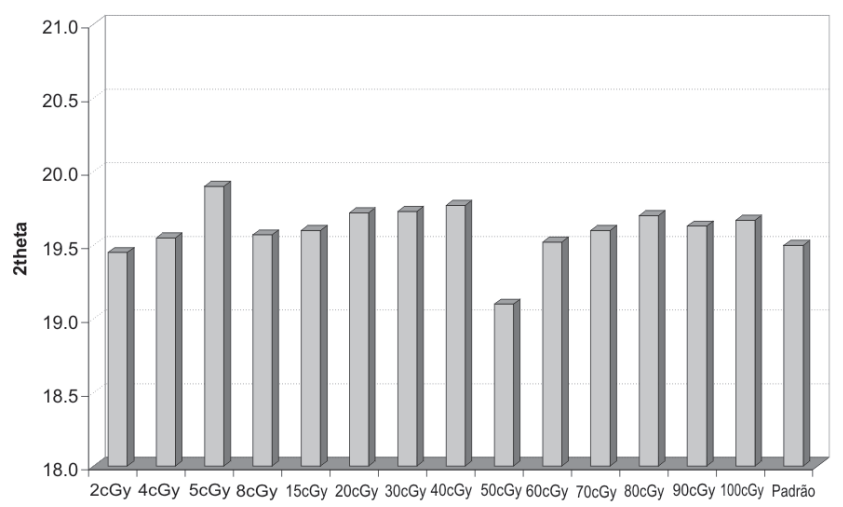

Figura 9. Variação de $\theta_{2}$ em função da dose para as amostras de PL

Pode-se observar a presença de dois picos estreitos em $28,37^{\circ} \pm 0,03^{\circ}$ e $31,7^{\circ} \pm 0,05^{\circ}$ no perfil de espalhamento típico obtido para as amostras de plasma (Figura 3). Esses picos são devidos à presença de cristais de potássio presentes no plasma. A análise apresentada nesse trabalho foi baseada apenas nos dois picos principais largos.

Os valores médios de $\theta_{1}$ e $\theta_{2}$ foram comparados com aqueles medidos a partir dos perfis de difração obtidos para as amostras de ST, MC e PLirradiadas com 2, 4, 5, 8, 15, 20, 30, 40, 
$50,60,70,80,90$ e 100 cGy. As figuras 4 e 6 mostram a variação da posição angular do primeiro pico de difração das amostras de ST, MC e PL em função da dose, respectivamente.

As figuras 7 a 9 mostram a variação da posição angular do segundo pico de difração em função da dose para as amostras de ST, MC e PL, respectivamente.

\section{Conclusões}

Os valores de $\theta_{1}$ e $\theta_{2}$ obtidos nas amostras de plasma irradiados são maiores que os obtidos para as amostras de plasma não irradiado. Para as amostras de sangue total irradiado nas doses de 80, 90 e $100 \mathrm{cGy}$ (para $\theta_{1}$ ) e nas doses de 8 , 40 e $50 \mathrm{cGy}$ (para $\theta_{2}$ ) o mesmo comportamento foi observado.

Para as amostras de matriz celular, todos os valores de $\theta_{1}$ e $\theta_{2}$ encontrados apresentaram valor menor que os valores obtidos pela amostra padrão.

Pode-se perceber que a forma das assinaturas do sangue total e matriz celular é bastante semelhante. O perfil de espalhamento típico do plasma se distinguiu dos demais pelas diferenças observadas na posição angular dos dois picos largos e pela presença de dois picos estreitos pronunciados.

O dano biológico pode ser causado por uma pequena dose de radiação; o conhecimento da dose em adição com as mudanças nos indicadores biológicos torna-se um outro agente importante no estudo dos riscos associados à radiação. Esse estudo permitiu observar as possíveis alterações estruturais nas amostras de sangue irradiado com baixas doses.

\footnotetext{
Abstract

The increase in ionizing radiation (IR) applications can expose people and objects to radiation fields, where the intensity depends on the type of source and the activity. In this work, scattering profiles of lyophilized samples of plasma, cellular matrix and total blood were studied. The scattering profiles were obtained using a Shiamadzu $X$-Ray diffractometer (XRD 6000) at the Laboratorio de Instrumentação Nuclear, LIN/Coppe. The samples were irradiated with doses of from 2cGy to 100cGy at Inca in Rio de Janeiro and all the samples of plasma, cellular matrix and total blood were lyophilized at $-60^{\circ} \mathrm{C}$ and $780 \mathrm{mmHg}$ until the water was totally eliminated. Scanning involved scattering angles of from $5^{\circ}$ to $35^{\circ}$ in steps of $0.05^{\circ}$ with an integration time of 3 seconds. The scattering profiles of the irradiated samples were compared with non-irradiated sample profiles. In this study it was observed that the cellular matrix and total blood sample profiles are similar. The scattering profile of plasma is very characteristic because in addition to the first and second peaks, these samples show two other narrow peaks. The 1 and 2 values of irradiated plasma samples are greater than nonirradiated ones and the 1 and 2 values of the irradiated cellular matrix samples are smaller than ones obtained using non-irradiated cellular matrix samples. Rev. bras. hematol. hemoter. 2008; 30(2): 132-135.
}

Key words: Blood; X-ray diffraction.

\section{Agradecimentos}

Os autores agradecem à agência brasileira Capes pelo suporte financeiro e ao Laboratório de Análises Clínicas Dr. Eliel Figueiredo.

\section{Referências Bibliográficas}

1. Evans SH, Bradley DA, Dance DR, Bateman JE, Jones CH. Measurement of small-angle photon scattering for some breast tissues and tissue substitute materials. Phys Med Biol. 1991;36 (1):7-18 .

2. Elshemey WM, Elsayed AA, El-Lakkani A. Characteristics of lowangle x-ray scattering from some biological samples. Phys Med Biol. 1999;44(12):2907-15.

3. Desouky OS, Elshemey WM, Selim NS, Ashour AH. Analysis of lowangle $\mathrm{x}$-ray scattering peaks from lyophilized biological samples. Phys Med Biol. 2001;46(8):2099-106.

4. Castro CRF, Barroso RC, Anjos MJ, Lopes RT, Braza D et al. Coherent scattering characteristics of normal and pathological breast human tissues. Radiation Physics and Chemistry. 2004;71(3-4):649-51.

5. Castro CRF, Barroso RC, Lopes RT. Scattering signatures for some human tissues using synchrotron radiation. X-ray Spectrometry. 2005;34(6):477-80.

6. Elshemey WM, Desouky OS, Ashour Ah et al. Low-angle X-ray scattering from lyophilized blood constituents. Phys Med Biol. 2001;46(2):531-9.

7. Barroso RC, Lopes RT, Gonçalves OD, de Jesus EF. Angle-dispersive diffraction with synchrotron radiation at Laboratório Nacional de Luz Síncrotron (Brazil): potential for use in biomedical imaging.. Appl. Radiat. Isoto. 2000;53(4-5):717-24.

Avaliação: Editor e dois revisores externos Conflito de interesse: não declarado

Recebido: 10/03/2007

Aceito: 11/04/2007 